Provided for non-commercial research and education use. Not for reproduction, distribution or commercial use.

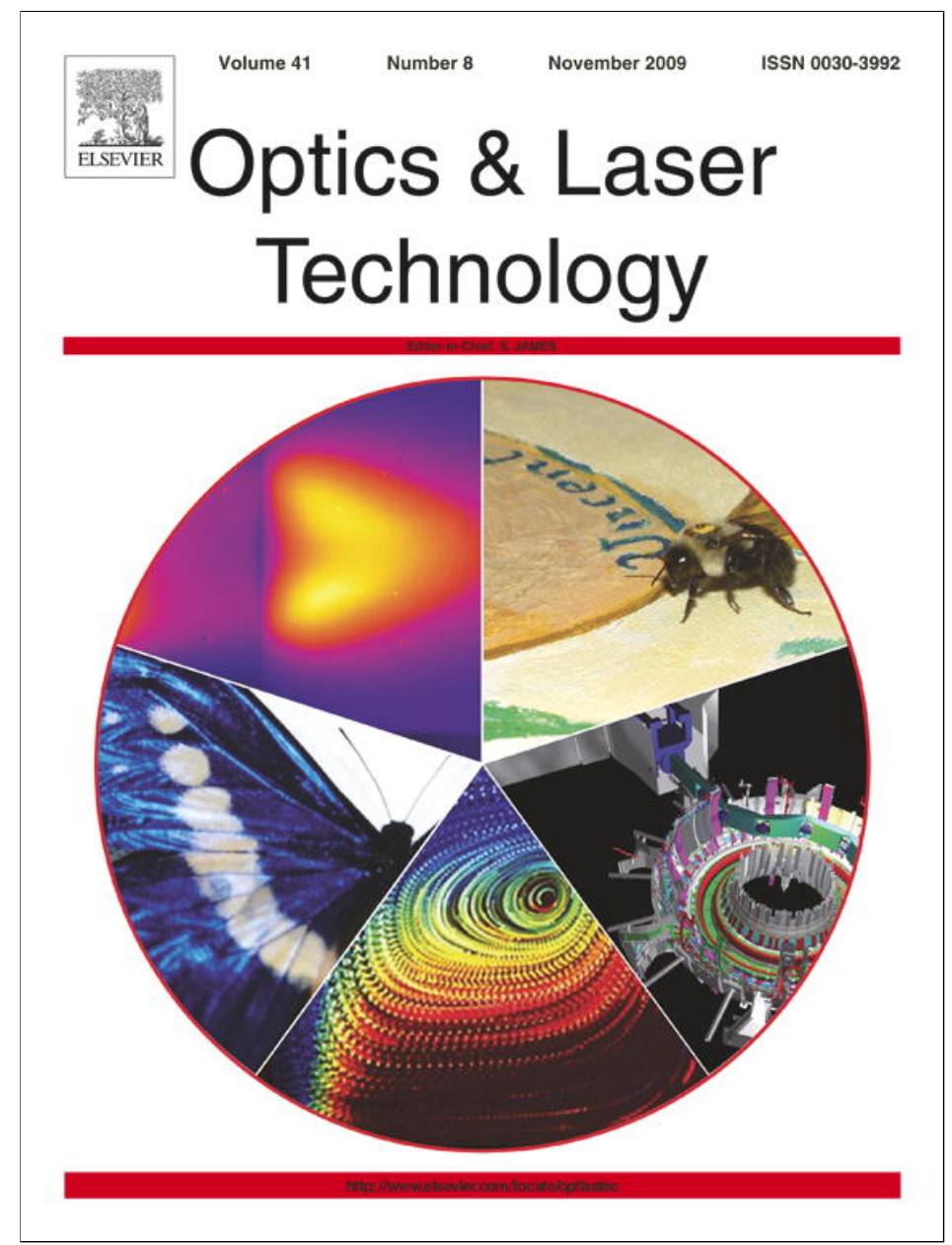

This article appeared in a journal published by Elsevier. The attached copy is furnished to the author for internal non-commercial research and education use, including for instruction at the authors institution and sharing with colleagues.

Other uses, including reproduction and distribution, or selling or licensing copies, or posting to personal, institutional or third party websites are prohibited.

In most cases authors are permitted to post their version of the article (e.g. in Word or Tex form) to their personal website or institutional repository. Authors requiring further information regarding Elsevier's archiving and manuscript policies are encouraged to visit:

http://www.elsevier.com/copyright 


\title{
Effects of experimental parameters on elemental analysis of coal by laser-induced breakdown spectroscopy
}

\author{
Jie $\mathrm{Li}^{\mathrm{a}}$, Jidong $\mathrm{Lu}^{\mathrm{a}}{ }^{\mathrm{a}}$, Zhaoxiang Lin ${ }^{\mathrm{b}}$, Shunsheng Gong ${ }^{\mathrm{c}}$, Chengli Xie ${ }^{\mathrm{a}}$, Liang Chang ${ }^{\mathrm{b}}$, \\ Lifei Yang ${ }^{\mathrm{a}}$, Pengyan $\mathrm{Li}^{\mathrm{a}}$ \\ a State Key Laboratory of Coal Combustion, Huazhong University of Science and Technology, Wuhan 430074, China \\ b Laboratory for Laser Spectrum Research, South-Central University for Nationalities, Wuhan 430074, China \\ ${ }^{\mathrm{c}}$ Wuhan Institute of Physics and Mathematics, Chinese Academy of Sciences, Wuhan 430071, China
}

\section{A R T I C L E I N F O}

\section{Article history:}

Received 2 November 2008

Received in revised form

22 February 2009

Accepted 9 March 2009

Available online 9 May 2009

\section{Keywords:}

Laser-induced breakdown spectroscopy

Coal

Experimental parameter

\begin{abstract}
A B S T R A C T
The purpose of this work is to improve the precision of the elemental analysis of coal using laserinduced breakdown spectroscopy (LIBS). The LIBS technique has the ability to allow simultaneous elemental analysis and on-line determination, so it could be used in the elemental analysis of coal. Organic components such as C, H, O, N and inorganic components such as $\mathrm{Ca}, \mathrm{Mg}, \mathrm{Fe}, \mathrm{Al}, \mathrm{Si}, \mathrm{Ti}, \mathrm{Na}$, and $\mathrm{K}$ of coal have been identified. The precision of the LIBS technique depends strongly on the experimental conditions, and the choice of experimental parameters should be aimed at optimizing the repeatability of the measurements. The dependences of the relative standard deviation (RSD) of the LIBS measurements on the experimental parameters including the sample preparation parameters, lensto-sample distance, sample operation mode, and ambient gas have been investigated. The results indicate that the precision of LIBS measurements for the coal sample can be improved by using the optimum experimental parameters.
\end{abstract}

(c) 2009 Elsevier Ltd. All rights reserved.

\section{Introduction}

Laser-induced breakdown spectroscopy (LIBS) is a laser based, rapid and sensitive optical diagnostic technique used to detect certain atomic species. The foundation for LIBS is a solid state, short-pulsed laser that is focused on a sample to generate a hightemperature plasma. When the plasma cools, the excited atomic, ionic, and molecular fragments produced within the plasma emit radiation that is characteristic of the elemental composition of the material in the volatilized sample. The LIBS technique has been used extensively for analysis of elemental concentrations in a variety of solid [1], liquid [2], gas [3], and aerosol [4] samples.

Simultaneous elemental analysis is required in the assessment of the quality of coal. Laser-induced breakdown spectroscopy has ability to allow simultaneous determination and detect the lowatomic number elements, so it could be used in the on-line elemental analysis of coal. Many researchers have investigated the analysis of coal by means of laser-induced breakdown spectroscopy [5-15]. The coal sample form can be lump, powder or pellet. Zhang et al. [15] used a powder coal sample, but more researchers such as Wallis [8] and Mateo [12] have used the pellet coal sample

\footnotetext{
* Corresponding author.

E-mail address: jdlu@mail.hust.edu.cn (J. Lu).
}

for the LIBS measurements. The characteristics of the laserinduced plasmas may be different for different coal sample forms. The different coal sample forms influence the precision of LIBS measurements in different ways. A pellet coal sample is frequently used, but it differed from the pellet sample made from a homogeneous mixture. Many investigations have been undertaken on the effects of experimental parameters on alloy samples or homogeneous pellets [16,17]. It is difficult to guarantee precise and accurate quantitative results in LIBS measurements, especially for the heterogeneous materials such as coal. As far as we know, there are no papers that consider the effects of the experimental parameters on the precision of coal analysis by LIBS.

The focus of the present paper is to identify and quantify the variables that affect the precision of LIBS measurements for pellet coal samples. Important analysis parameters which influence the precision of coal analysis by LIBS include the sample preparation parameters, lens-to-sample distance (LTSD), sample operation mode, and ambient gas. These parameters have been investigated in this study and it is shown that their control can improve the precision of LIBS measurements. Furthermore, the calibration is closely related to the accuracy which is also important for LIBS analysis. The calibration curves of the coal sample are given in this paper to allow the comparison between the LIBS analytical results and the certified analytical results. 


\section{Experimental}

A schematic diagram of the experimental set-up is shown in Fig. 1. A pulsed Q-switched Nd:YAG laser (Spectra-Physics, model Pro 290) is used to generate the plasma. The laser operates at the $1064 \mathrm{~nm}$ with $10 \mathrm{~ns}$ pulse width and $600 \mathrm{~mJ}$ pulse energy. The laser energy is reduced to approximately $30 \mathrm{~mJ}$ by means of an optical attenuator (Beamtech), consisting in a half-wave plate that rotates the polarization plane of the laser light and a polarizing beamsplitter cube. The laser beam is focused on the sample via a lens with $195 \mathrm{~mm}$ focal length. The sample is positioned on a rotation stage. The power density (calculated at the focal length of the lens) on the sample is on the order of $10^{10} \mathrm{~W} / \mathrm{cm}^{2}$.

The light emitted by the plasma is collected by an optical collector, and is fed to the slit of an Echelle spectrograph (Andor, model Mechelle 5000) through a 2-m-long optical fiber. The spectrograph provides a constant spectral resolution $(\lambda / \Delta \lambda)$ of 4000 over a wavelength range $200-900 \mathrm{~nm}$. The plasma dispersed light is detected by using a gated, intensified charge-coupled device (ICCD), Andor, model DH 734. The ICCD consists of $1024 \times 1024$ elements, the size of the detector chip is $13 \mathrm{~mm} \times 13$ $\mathrm{mm}$ and the spectral response range is $185-850 \mathrm{~nm}$. The ICCD intensifier has a diameter of $18 \mathrm{~mm}$, ensuring that all pixels of the array are active. Before the experiment, a wavelength calibration using an Hg lamp (Ocean Optics, model HG-1) and a spectral response calibration using a deuterium-halogen lamp (Avantes, model AvaLight-DH-CAL) are carried out. A digital delay/pulse generator (Stanford Research Systems, model DG535) is used, and the Q-switch is triggered by the DG535 synchronized to the data acquisition system controlled by a computer. An optimum delay time of $0.5 \mu$ s was selected to avoid the continuum emission. A gate width of $2 \mu$ s was used which collected a large fraction of the elemental emission. Fifty summed spectra comprised one measurement, which was then repeated five times.

One bituminous coal sample from Pingxiang mine in China was received and used in this study. The coal sample was analyzed by a certified chemical analysis method before the LIBS experiments were undertaken.

The sample preparation parameters, lens-to-sample distance, sample operation mode, and ambient gas can affect coal analysis by LIBS. In this study, these parameters were systematically changed and their effects on the repeatability were studied. The results of repeated measurements were used to determine the repeatability of LIBS measurement. The relative standard deviation (\%RSD) was used to evaluate the repeatability, and it can be computed as

$\% \operatorname{RSD}=100 \% \times\left[\sum\left(x_{i}-M\right)^{2} /(n-1)\right]^{1 / 2} / M$

where $n$ is the number of a set of measurements; $x_{i}$ is the result of each measurement; $M$ is the arithmetic mean value of the set of repeated measurements.

\section{Results and discussion}

\subsection{Spectrum analysis}

The basis of LIBS measurement is the analysis of the plasma spectrum which contains information about the elements in the sample. This information is in the form of emission lines, located at specific wavelengths [18]. The emission spectra of coal obtained by laser-induced breakdown spectroscopy were recorded. A representative spectrum of coal in the $240-850 \mathrm{~nm}$ spectral region is shown in Fig. 2, where the main peaks were identified using the National Institute of Standards and Technology (NIST) electronic database [19]. This spectrum shows a high density of emission lines resulting from the complex composition of coal, and the spectrum reveals the key inorganic components of coal such as $\mathrm{Ca}, \mathrm{Mg}, \mathrm{Fe}, \mathrm{Al}, \mathrm{Si}, \mathrm{Ti}, \mathrm{Na}$, and $\mathrm{K}$. In addition, organic

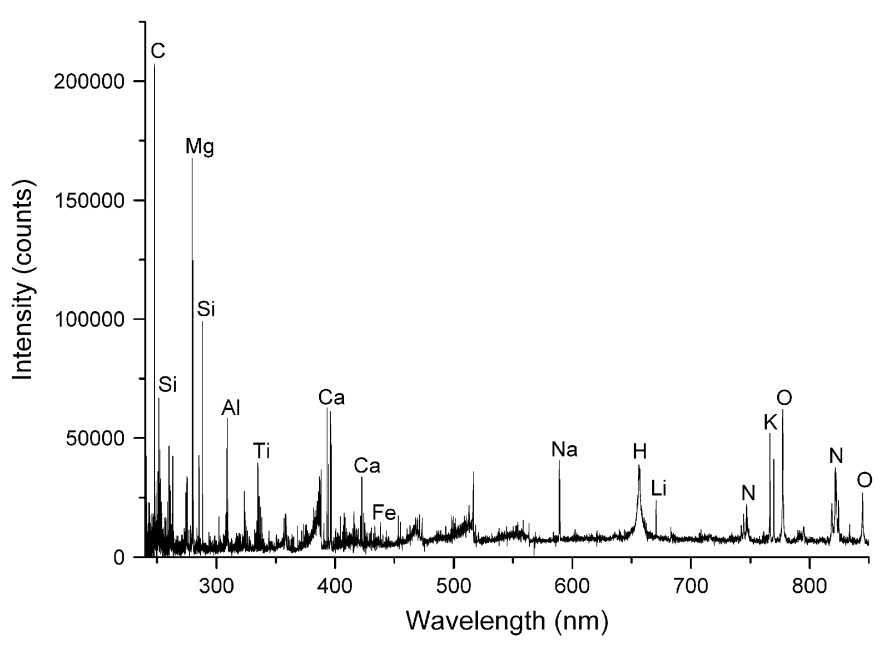

Fig. 2. Emission spectrum of the coal sample by laser-induced breakdown spectroscopy.

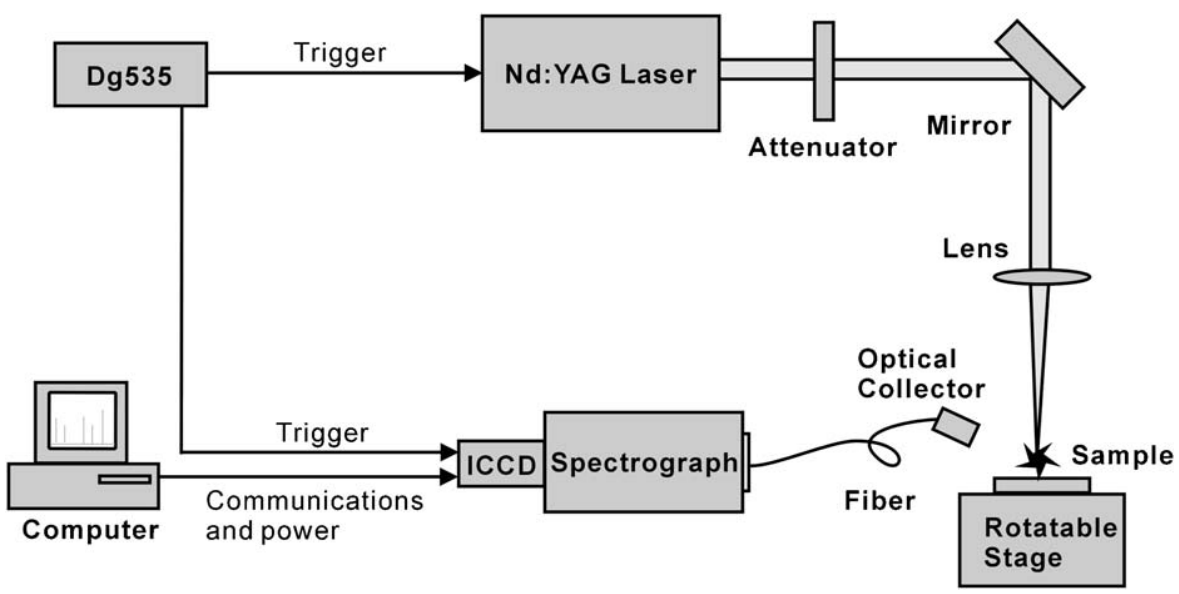

Fig. 1. LIBS experimental set-up. 
Table 1

List of some stronger element emission lines observed coal spectrum.

\begin{tabular}{ll}
\hline Element & Emission line $(\mathrm{nm})$ \\
\hline $\mathrm{C}$ & 247.9 \\
$\mathrm{H}$ & 656.3 \\
$\mathrm{O}$ & $777.4,844.6$ \\
$\mathrm{~N}$ & $742.4,744.2,746.8,821.6$ \\
$\mathrm{Ca}$ & $315.9,317.9,393.4,396.9,422.7$ \\
$\mathrm{Al}$ & $308.2,309.3,394.4,396.2$ \\
$\mathrm{Fe}$ & $274.9,275.6,404.6,438.4$ \\
$\mathrm{Mg}$ & $279.6,280.3,285.2$ \\
$\mathrm{Si}$ & $251.6,263.1,288.2,390.6$ \\
$\mathrm{Ti}$ & $323.5,334.9,336.1,337.3,338.4$ \\
$\mathrm{Na}$ & $589.0,589.6$ \\
$\mathrm{~K}$ & $766.5,769.9$ \\
\hline
\end{tabular}

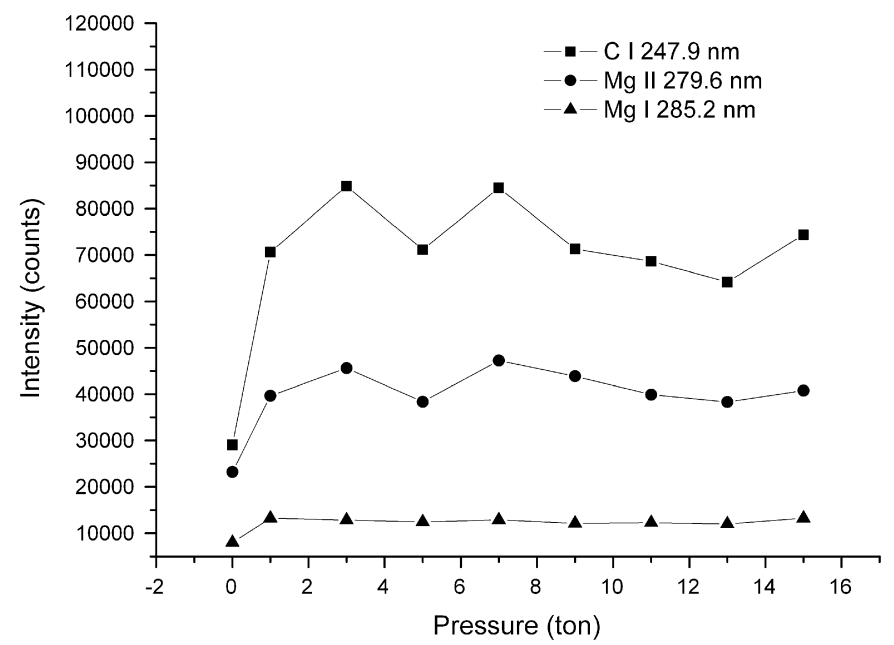

Fig. 3. Variations of the emission line intensities with the change of coal samples (powder sample and pellet samples).

components such as $\mathrm{C}, \mathrm{H}, \mathrm{O}$, and $\mathrm{N}$ are also clearly seen. Most elements in the coal have more than one line, and Table 1 lists some of the stronger emission lines observed, most of which are commonly found in LIBS spectra of coal. The C I $247.9 \mathrm{~nm}, \mathrm{Mg}$ II $279.6 \mathrm{~nm}$, Mg I $285.2 \mathrm{~nm}$, Al I $309.3 \mathrm{~nm}$, Ca II $393.4 \mathrm{~nm}$, Ca I $422.7 \mathrm{~nm}$, and O I $777.4 \mathrm{~nm}$ emission lines have been selected for analysis because they are intense and have minimal interference from other emission lines. The intensities of emission lines are determined by using the peak height.

\subsection{Sample preparation parameters}

The powder coal sample and pellet coal samples with different pressing force were investigated in this experiment in order to compare the repeatability for the LIBS measurements. A portion of powder coal was transferred into a steel dish (13 $\mathrm{mm}$ diameter) and compacted into a pellet, with the use of a certain force for $1 \mathrm{~min}$. The pressing forces were $1,3,5,7,9,11,13$, and $15 \mathrm{t}$, respectively. The results of the line intensities and their RSD of the C I $247.9 \mathrm{~nm}, \mathrm{Mg}$ II $279.6 \mathrm{~nm}$, and $\mathrm{Mg}$ I $285.2 \mathrm{~nm}$ emission lines obtained from the powder coal sample and pellet coal samples are shown in Figs. 3 and 4, respectively. The " 0 " positions in the figures correspond to the powder coal sample. As can be seen, the line intensities of pellet coal samples are on a level and about three times for C I $247.9 \mathrm{~nm}$ and two times for Mg II $279.6 \mathrm{~nm}$ and $\mathrm{Mg}$ I $285.2 \mathrm{~nm}$ more than the powder coal sample. It is apparent that the RSD of the powder sample are larger than $16 \%$, and this

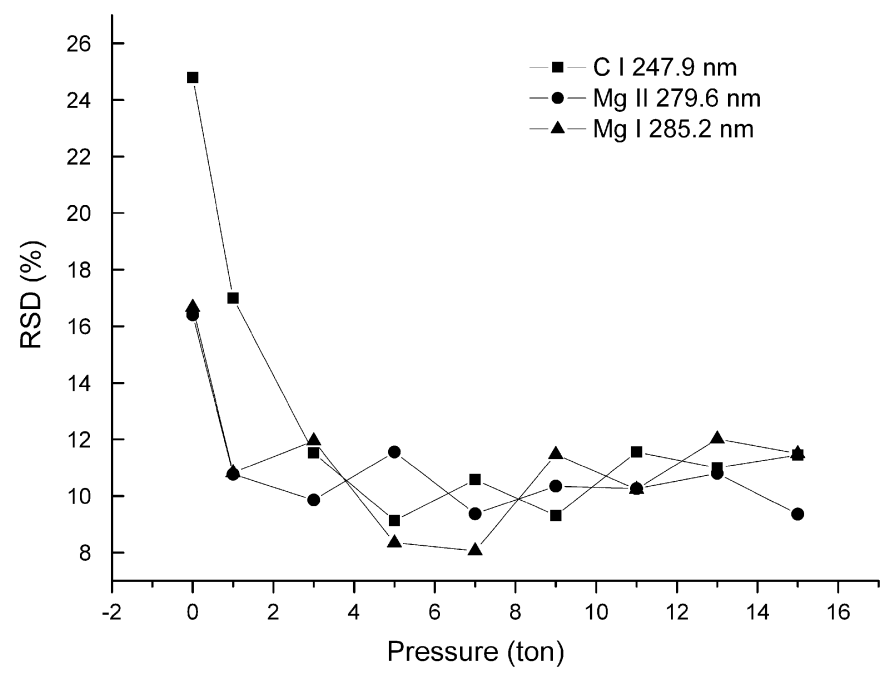

Fig. 4. Variations of the RSD of the emission line intensities with the change of coal samples (powder sample and pellet samples).

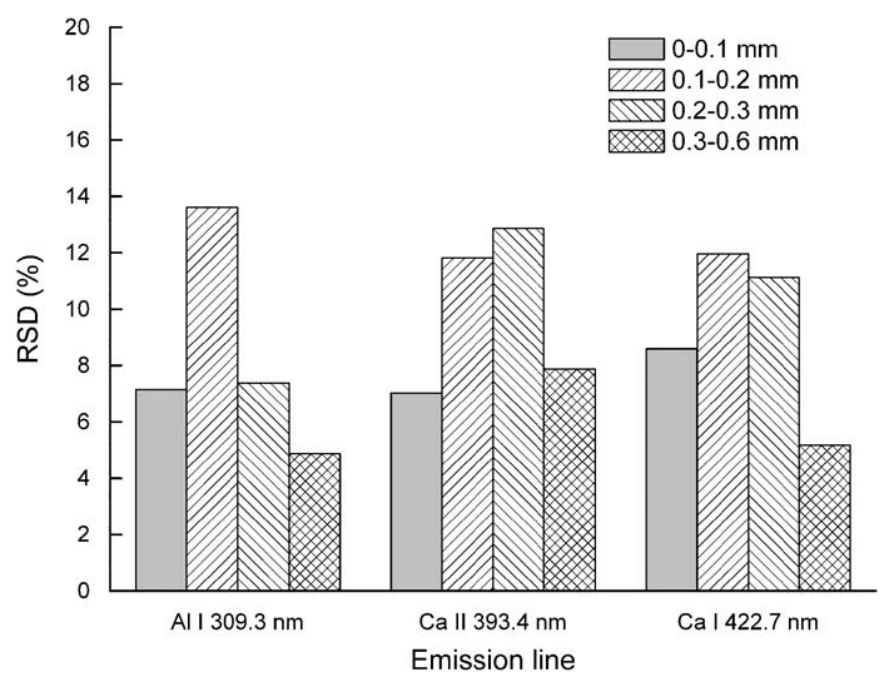

Fig. 5. RSD of the emission lines intensities for different particle sizes of the coal sample.

may be caused by the persistent aerosols and relatively bigger crater formation [20]. The RSD are almost constant and about $10 \%$ when the pressing force is greater than $3 \mathrm{t}$. For the powder coal sample, the structure is loose and the surface is irregular. The ablated mass may be different pulse-to-pulse, and the particulates easily escape to the atmosphere, which can intercept the laser beam and influence the plasma formation. The pressing procedure produces uniform dense pellets with an even, smooth analytical surface exposed to the laser beam [8]. In the next experiment, pellet coal samples with $10 \mathrm{t}$ pressure were used.

Particle size is a physical factor which can affect LIBS analysis when analyzing a powder sample. Coal particles of four different size groups $(0-0.1,0.1-0.2,0.2-0.3$, and $0.3-0.6 \mathrm{~mm})$ were deposited on filters and analyzed by LIBS. The RSD of emission line intensities for each particle size group are shown in Fig. 5 . The RSD for the particle size of $0-0.1$ or $0.3-0.6 \mathrm{~mm}$ is small. There may be two influencing factors: one is the intermolecular force, the other is the characteristics of the surface. For the smaller particle size, the intermolecular force between the particles is bigger and the pellet coal sample is rigid, so the plasmas are stable. For the larger particle size, the surface area of each particle 
Table 2

RSD (\%) of the emission lines intensities of the coal samples of as-received basis and air-dried basis.

\begin{tabular}{lcc}
\hline Emission line (nm) & As-received basis (ar) & Air-dried basis (ad) \\
\hline C I 247.9 & 7.67 & 13.67 \\
Mg II 279.6 & 10.61 & 8.52 \\
Mg I 285.2 & 14.72 & 11.62 \\
Al I 309.3 & 8.22 & 11.37 \\
Ca II 393.4 & 5.18 & 5.18 \\
Ca I 422.7 & 8.20 & 6.09 \\
\hline
\end{tabular}

is greater, and most of the laser energy is focused on the surface rather that ob the gaps. However, it is found that the pellet samples made from coal of large particle size are fragile. Hence, the coal of small particle size $(0-0.1 \mathrm{~mm})$ is chosen for this study.

The moisture content is an important factor in the coal sample, so it is considered in this study too. "As-received" and "air-dried" coal samples which have different moisture content were compared by LIBS. The RSD of the line intensities are shown in Table 2. As can be seen, there is no obvious difference between the "as-received" and "air-dried" coal samples. However, the content in the air-dried coal sample is constant in the library, and the coal sample of as-received sample is the reverse. Therefore, the airdried coal sample is used in this study.

\subsection{Lens-to-sample distance}

In LIBS, a focused laser pulse is used to ablate material from a surface and to form laser plasma that excites the vaporized material. Geometric factors, such as the lens-to-sample distance can greatly influence the LIBS analytical results [21]. Hence, the LTSD must be considered for the purpose of obtaining better quantitative results. Since the LTSD is an important parameter that affects LIBS analysis, some researchers have investigated its effect on the spectra [22]. The power density incident on the sample is a strong function of the lens-to-sample distance and the power density determines, in part, emission lines intensities and the mass of sample ablated [18]. For the sake of convenience, the position of the focal point is used instead of the lens-to-sample distance. There are three situations: the position of the focal point is above, on or inside the sample. It was found that when the position of the focal point was above the surface, the phenomenon of air breakdown always occurred, leading to poor shot-to-shot repeatability, so this condition was not used.

The dependence of the emission lines intensities on the focal position is shown in Fig. 6. The ' 0 ' position of the focal spot corresponds to that position of the focusing lens where the laser pulse is focused on the surface of the sample, whereas negative numbers indicate the position of the focal spot inside the sample. The emission lines intensities are maxima when the laser is focused on the sample surface, and decrease when the laser is focused inside the sample. The line intensity of $\mathrm{Mg}$ II $279.6 \mathrm{~nm}$ is about three times than Mg I $285.2 \mathrm{~nm}$ when the laser is focused at the surface, but is only two times than $\mathrm{Mg}$ I $285.2 \mathrm{~nm}$ when the laser is focused $5 \mathrm{~mm}$ inside the surface. Meanwhile, the line intensity of Ca II $393.4 \mathrm{~nm}$ is about one and a half times that of Ca I $422.7 \mathrm{~nm}$ when the laser is focused at the surface, but is slightly less than Ca I $422.7 \mathrm{~nm}$ when the laser is focused $5 \mathrm{~mm}$ inside the surface. These mean that the Mg II $279.6 \mathrm{~nm}$ line declined faster than the Mg I $285.2 \mathrm{~nm}$ and the Ca II $393.4 \mathrm{~nm}$ lines also declined faster than the Ca I $422.7 \mathrm{~nm}$ line when the laser spot moved inside the surface. The variations of the line intensities of $\mathrm{Mg}$ I and Mg II and also Ca I and Ca II must be considered when choosing the optimum lens-to-sample distance. The variation of the spot size results in changes in the process of material ablation and

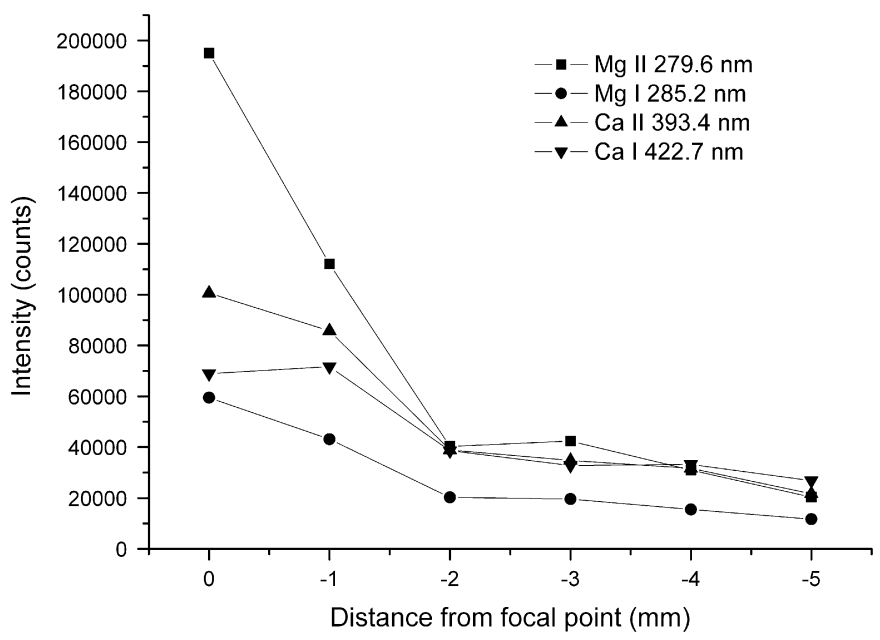

Fig. 6. Emission lines intensities as function of the position of the focal point.

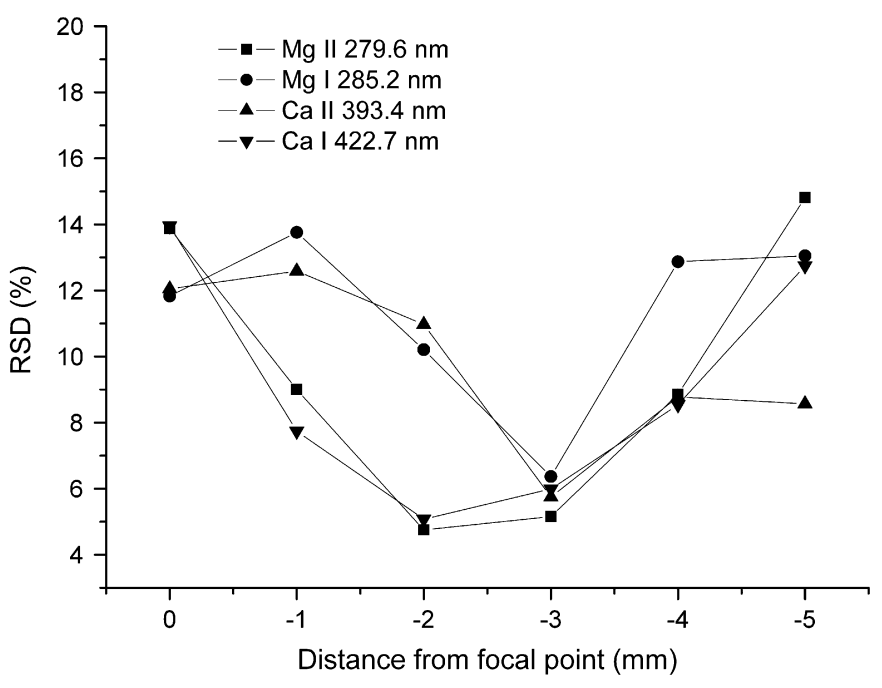

Fig. 7. RSD of the emission lines intensities as a function of position of the foca spot. The " 0 " position represents a focal spot on the surface, and value corresponds to a focal spot inside the surface of the sample.

plasma formation, which in turn affects the coupling of the laser energy to the sample.

Fig. 7 shows the variation of the RSD of the emission lines intensities with the change in the focal-spot position. As can be seen, the RSD are larger than 10\% when the laser is focused on the sample surface, indicating that the plasmas were not stable. Bassiotis et al. [23] thought this was due to the air breakdown, and that the plasma absorbed the laser light and thus only a part of it reached and ablated the target. The RSD of Mg II $279.6 \mathrm{~nm}$ and Ca I $422.7 \mathrm{~nm}$ are minima when the laser is focused $2 \mathrm{~mm}$ inside the surface, while the RSD of Mg I $285.2 \mathrm{~nm}$ and Ca II $393.4 \mathrm{~nm}$ are minima when the laser is focused $3 \mathrm{~mm}$ inside the surface. The increase in the RSD when the focal spot moved further inside the sample could be due to flatter and relatively colder plasma generated at the surface [17]. Hence, the focal spot of $3 \mathrm{~mm}$ inside the sample is chosen because the RSD are smaller than $10 \%$ for all the spectra lines in this situation. More to the point, for C I $247.9 \mathrm{~nm}$ emission line, the RSD was a minimum when the laser was focused on the sample surface, and increased slightly when the laser was focused inside the sample from 1 to $3 \mathrm{~mm}$, but increased quickly when the laser was focused further inside the sample. Aragon et al. [24] investigated the LTSD for the 
steel by LIBS. The C (I), Cr (II), Si (I), and Ni (II) emission lines were used, and the best LTSD values that the maximum of the intensities and the minimum RSD produced at a given pulse energy were different in some millimeters for the four analytical lines.

\subsection{Sample operation mode}

The uniformity of the sample surface can affect the LIBS analysis results. However, this effect may be decreased by using appropriate sample operation mode. In order to improve the precision, three modes of sample operation were compared in this study. In the first mode, 250 laser pulses were delivered at the same sample position. In the second mode, 50 laser pulses were delivered at the same sample position to produce a spectrum and 5 positions were used. In the third mode, the sample was rotating, thereby exposing a fresh position of sample to each laser pulse. The sample was also moved in the horizontal direction or changed for another one in order to ensure that the laser focused on a fresh position. The results of the RSD of the emission lines intensities obtained from three modes are shown in Fig. 8. It can be inferred from data in this figure that the different sample operation modes lead to different repeatability. The RSD are minima when the sample is in the third mode, this means that there is small change in the intensity. The RSD are maxima when the sample was in the first mode and this is due to the craters produced by the laser pulses. The size of the crater increased with time in this situation, and affected not only the sample ablation rate but also how the plasma imaged onto the optical fiber [17]. Hence, the third sample operation mode is chosen in order to enhance the precision of LIBS measurements. Some researchers, for example Body [9] and Salle [25], located the sample on a translation stage that moved the sample between laser pulses too.

\subsection{Ambient gas}

The ambient gas plays a key role in the development of the plasma and can change LIBS performance. In this study, ambient gases of air, nitrogen, and argon were used. Fig. 9 shows the line intensities obtained from these three ambient gases. As can be

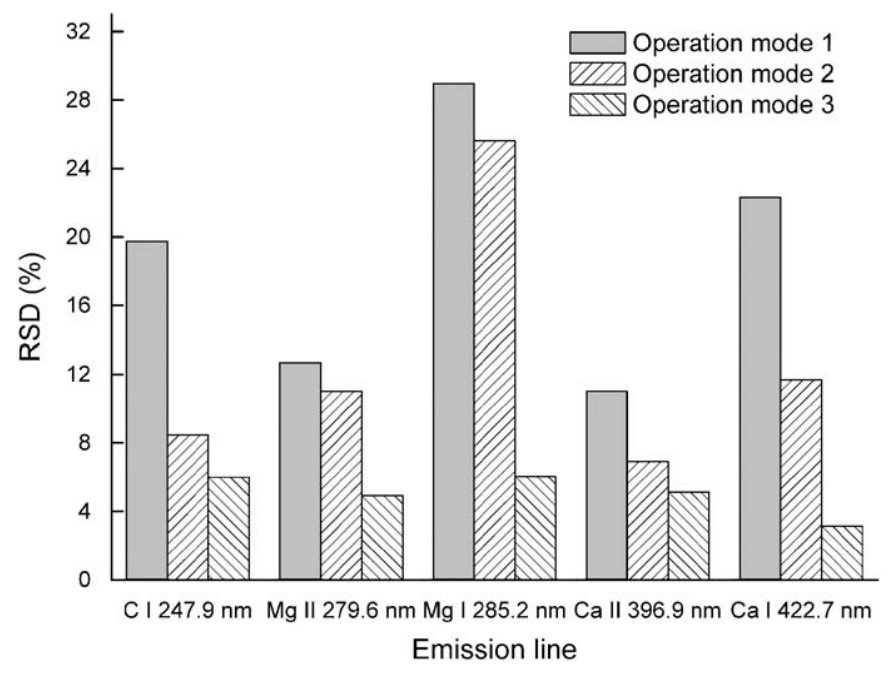

Fig. 8. RSD of the emission lines intensities as a function of sample operation mode. Mode 1: 250 laser pulses were delivered at the same sample position. Mode 2: 50 laser pulses were delivered at the same sample position to produce a spectrum and 5 positions were used. Mode 3: the sample was rotating, and 250 laser pulses were focused on the fresh position of the coal sample.

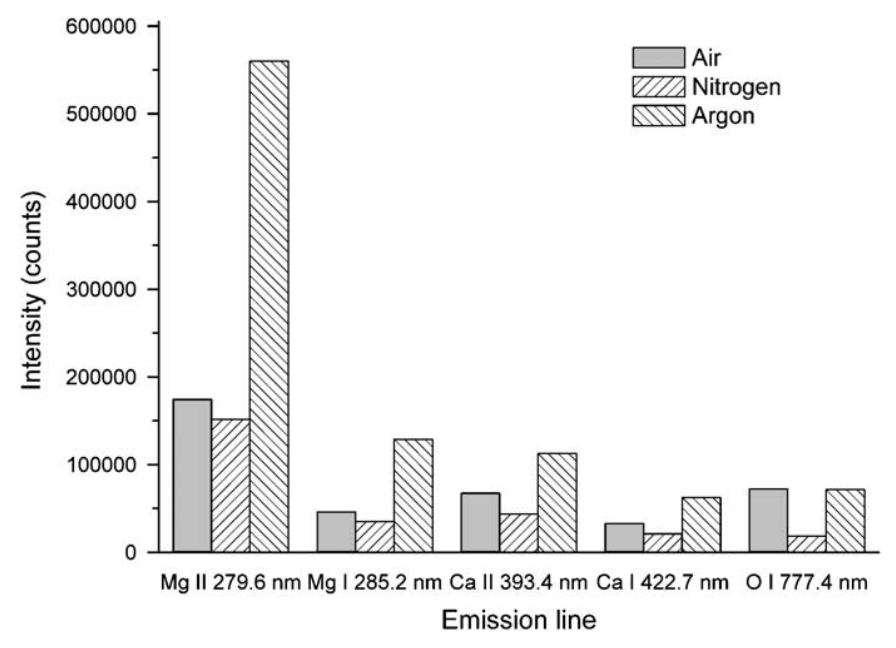

Fig. 9. Emission lines intensities in air, nitrogen, and argon.

Table 3

RSD (\%) of the emission lines intensities of the bituminous coal samples.

Emission line (nm) Pingxiang coal Wansheng coal Liupanshui coal Anping coal

\begin{tabular}{llllr}
\hline C I 247.9 & 6.00 & 9.57 & 4.99 & 4.10 \\
H I 656.3 & 5.36 & 4.45 & 4.85 & 6.50 \\
O I 777.4 & 5.83 & 8.22 & 4.48 & 8.83 \\
N I 746.8 & 5.89 & 7.04 & 4.64 & 12.48 \\
Ca II 393.4 & 5.73 & 6.85 & 8.13 & 6.76 \\
Ca I 422.7 & 3.14 & 5.55 & 8.54 & 8.46 \\
Al I 309.3 & 4.66 & 4.94 & 9.27 & 5.78 \\
Fe I 438.4 & 7.01 & 3.73 & 9.83 & 8.38 \\
Mg II 279.6 & 4.91 & 5.56 & 6.35 & 8.74 \\
Mg I 285.2 & 6.05 & 5.11 & 7.08 & 13.28 \\
Si I 288.2 & 4.35 & 7.80 & 7.40 & 10.09 \\
Ti II 334.9 & 7.52 & 9.11 & 7.48 & 15.04 \\
Na I 589.6 & 6.19 & 2.34 & 10.39 & 8.23 \\
K I 769.9 & 4.22 & 5.79 & 4.21 & 9.15 \\
\hline
\end{tabular}

seen, the line intensities are dependent on the ambient gas. The line intensities of Mg II $279.6 \mathrm{~nm}, \mathrm{Mg}$ I $285.2 \mathrm{~nm}$, Ca II $393.4 \mathrm{~nm}$, and Ca I $422.7 \mathrm{~nm}$ in air are slightly higher than in nitrogen. But the argon is demonstrated to produce the higher line intensities, and the line intensities are approximately three to four times than in air or in nitrogen.

It is worth mentioning that the line intensity of O I $777.4 \mathrm{~nm}$ in air is higher than that in nitrogen by a factor of about four. The air is mainly composed of nitrogen (approximately 78\%) and oxygen (approximately 21\%) with lesser amounts of other gases. The O I $777.4 \mathrm{~nm}$ spectral line in air is obtained from the $\mathrm{O}$ element both in the coal and the air.

\subsection{Evaluation}

In order to test the usefulness of the above parameter optimization results, the Pingxiang coal sample and three other coal samples from Wansheng, Liupanshui, and Anping mines in China were checked. The laser energy was $30 \mathrm{~mJ}$ and the laser was focused $3 \mathrm{~mm}$ inside the sample. The delay time was $0.5 \mu \mathrm{s}$ and the gate width was $2 \mu \mathrm{s}$. The pellet coal samples using $10 \mathrm{t}$ pressure were put on the rotation stage that moved the sample between the laser pulses. The plasma was generated in air at atmospheric pressure. Fifty summed spectra comprised one measurement, which was then repeated five times. The RSD of emission lines of organic components such as $\mathrm{C}, \mathrm{H}, \mathrm{O}, \mathrm{N}$ and 
a

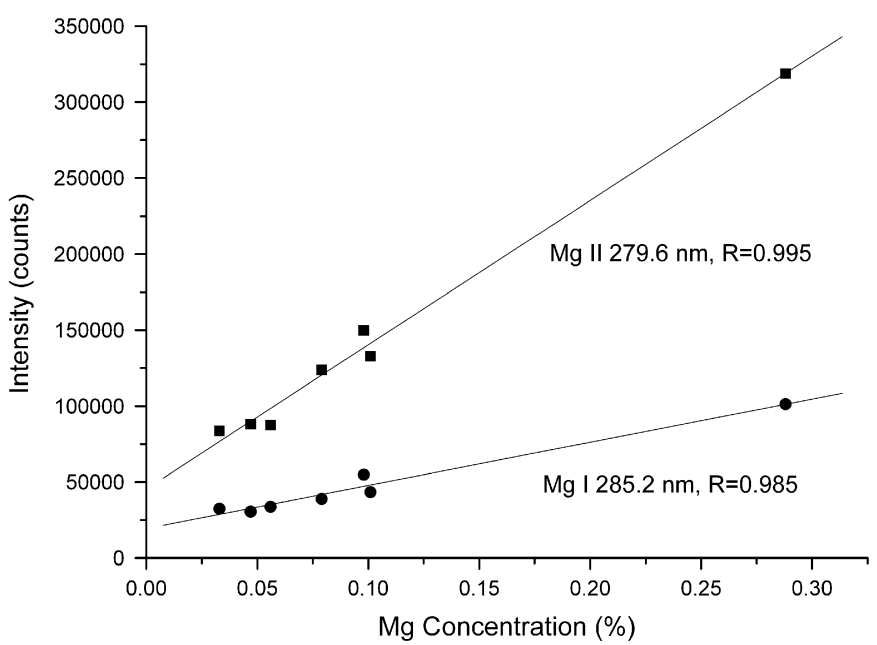

b

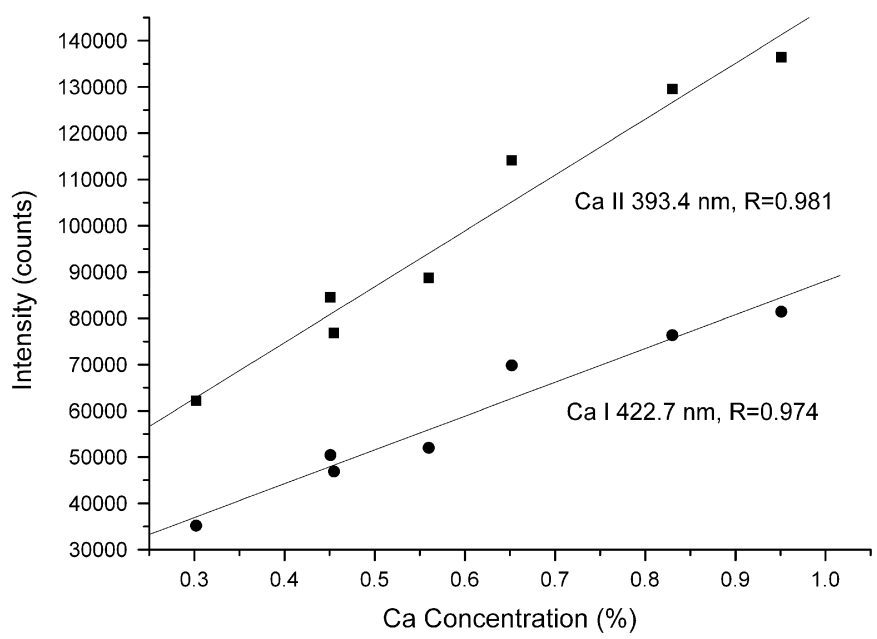

Fig. 10. Calibration curves for $\mathrm{Mg}$ and $\mathrm{Al}$ elements analyzed by LIBS: (a) Mg II $279.6 \mathrm{~nm}$ and $\mathrm{Mg}$ I $285.2 \mathrm{~nm}$, (b) Ca II $393.4 \mathrm{~nm}$ and Ca I $422.7 \mathrm{~nm}$. Linear correlation coefficients $(R)$ have been included in each graph.

inorganic components such as $\mathrm{Ca}, \mathrm{Mg}, \mathrm{Fe}, \mathrm{Al}, \mathrm{Si}, \mathrm{Ti}, \mathrm{Na}$, and $\mathrm{K}$ were calculated by using Eq. (1) mentioned above, and the results of RSD of four coal samples are summarized in Table 3. It is clear that most RSD of emission lines intensities are generally in the range $5-10 \%$ and these results are good.

Seven coal samples with a range of elemental concentrations were analyzed by LIBS for calibration. Calibration curves of the line intensities versus the known concentrations of the considered elements in the coal were obtained. It is to be observed that the calibration curves for organic elements $(\mathrm{C}, \mathrm{H}, \mathrm{O}$, and $\mathrm{N}$ ) were not good. However, calibration curves for inorganic elements were good, and Fig. 10 shows the calibration curves for the emission lines of Mg II $279.6 \mathrm{~nm}, \mathrm{Mg}$ I $285.2 \mathrm{~nm}$, Ca II $393.4 \mathrm{~nm}$, and Ca I $422.7 \mathrm{~nm}$. As can be seen, the calibration curves are linear over the range shown, and the linear correlation coefficients for $\mathrm{Mg}$ and $\mathrm{Ca}$ are high. The slope of the calibration curve of the Mg II $279.6 \mathrm{~nm}$ line is greater than that of the $\mathrm{Mg}$ I $285.2 \mathrm{~nm}$ line, and the slope of the calibration curve of the Ca II $393.4 \mathrm{~nm}$ line is greater than that of the Ca I $422.7 \mathrm{~nm}$ line. Clearly, as the slope increases, the sensitivity in the LIBS technique increases. Therefore, when the $\mathrm{Mg}$ and Ca concentrations are low the Mg II $279.6 \mathrm{~nm}$ and Ca II $393.4 \mathrm{~nm}$ lines are a better choice that the $\mathrm{Mg}$ I $285.2 \mathrm{~nm}$ and Ca I $422.7 \mathrm{~nm}$ lines.

\section{Conclusions}

A LIBS set-up using an Echelle spectrograph equipped with an ICCD detector for simultaneous analysis of the coal sample has been established. The detected elements in the LIBS spectra included the principal coal components such as $\mathrm{C}, \mathrm{H}, \mathrm{O}$, and $\mathrm{N}$ in addition to inorganic components such as $\mathrm{Ca}, \mathrm{Mg}, \mathrm{Fe}, \mathrm{Al}, \mathrm{Si}, \mathrm{Ti}, \mathrm{Na}$, and $\mathrm{K}$. The experimental parameters such as the sample preparation parameters, lens-to-sample distance, sample operation mode, and ambient gas have been investigated, and the optimum parameters which improved the precision of LIBS measurement have been obtained. The RSD of the emission lines intensities obtained from the elements of coal samples are smaller than $10 \%$ by using the parameters given in this paper. Calibration curves for inorganic elements such as $\mathrm{Mg}$ and $\mathrm{Ca}$ elements have been given, and the linear correlation coefficients are high. It has been shown that the precision of elemental analysis of coal by LIBS can be improved by using the proper experimental parameters.

\section{Acknowledgment}

This work is supported by the National Natural Science Foundation of China (No.50576029, No.50846041).

\section{References}

[1] Capitelli F, Colao F, Provenzano MR, Fantoni R, Brunetti G, Senesi N. Determination of heavy metals in soils by laser induced breakdown spectroscopy. Geoderma 2002;106:45-62.

[2] Lazic V, Colao F, Fantoni R, Spizzicchino V. Laser-induced breakdown spectroscopy in water: improvement of the detection threshold by signal processing. Spectrochimica Acta Part B 2005;60:1002-13.

[3] Gamala YE, Omar MM. Study of the electron kinetic processes in laserinduced breakdown of electronegative gases over an extended wavelength range. Radiation Physics and Chemistry 2001;62:361-70.

[4] Yoshiie R, Yamamoto Y, Uemiya S, Kambara S, Moritomi H. Simple and rapid analysis of heavy metals in sub-micron particulates in flue gas. Powder Technology 2008;180:135-9.

[5] Ottesen DK, Baxter LL, Radziemski LJ, Burrows JF. Laser spark emission spectroscopy for in situ, real-time monitoring of pulverized coal particle composition. Energy \& Fuels 1991;5:304-12.

[6] Zhang H, Singh JP, Yueh F, Cook RL. Laser-induced breakdown spectra in a coal-fired MHD facility. Applied Spectroscopy 1995;49:1617-23.

[7] Kurihara M, Ikeda K, Izawa Y, Deguchi Y, Tarui H. Optimal boiler control through real-time monitoring of unburned carbon in fly ash by laser-induced breakdown spectroscopy. Applied Optics 2003;42:6159-65.

[8] Wallis FJ, Chadwick BL, Morrison RJS. Analysis of lignite using laser-induced breakdown spectroscopy. Applied Spectroscopy 2000;54:1231-5.

[9] Body D, Chadwick BL. Simultaneous elemental analysis system using laser induced breakdown spectroscopy. Review of Scientific Instruments 2001;72:1625-9.

[10] Chadwick BL, Body D. Development and commercial evaluation of laserinduced breakdown spectroscopy chemical analysis technology in the coal power generation industry. Applied Spectroscopy 2002;56:70-4.

[11] Body D, Chadwick BL. Optimization of the spectral data processing in a LIBS simultaneous elemental analysis system. Spectrochimica Acta Part B 2001;56:725-36

[12] Mateo MP, Nicolas G, Yanez A. Characterization of inorganic species in coal by laser-induced breakdown spectroscopy using UV and IR radiations. Applied Surface Science 2007;254:868-72.

[13] Gaft M, Sapir-Sofer I, Modiano H, Stana R. Laser induced breakdown spectroscopy for bulk minerals online analyses. Spectrochimica Acta Part B 2007;62:1496-503.

[14] Gaft M, Dvir E, Modiano H, Schone U. Laser induced breakdown spectroscopy machine for online ash analyses in coal. Spectrochimica Acta Part B 2008;63:1177-82.

[15] Zhang L, Dong L, Dou H, Yin W, Jia S. Laser-induced breakdown spectroscopy for determination of the organic oxygen content in anthracite coal under atmospheric conditions. Applied Spectroscopy 2008;62:458-63.

[16] Castle BC, Talabardon K, Smith BW, Winefordner JD. Variables influencing the precision of laser-induced breakdown spectroscopy measurements. Applied Spectroscopy 1998;52:649-57.

[17] Lai B, Zheng H, Yueh FY, Singh JP. Parametric study of pellets for elemental analysis with laser-induced breakdown spectroscopy. Applied Optics 2004;43:2792-7. 
[18] Cremers DA, Radziemski LJ. Handbook of laser-induced breakdown spectroscopy. New York: Wiley; 2006.

[19] NIST electronic database, at $\langle$ http://physics.nist.gov/PhysRefData $\rangle$.

[20] Wisbrun R, Schechter I, Niessner R, Schroder H, Kompa KL. Detector for trace elemental analysis of solid environmental samples by laser plasma spectroscopy. Analytical Chemistry 1994;66:2964-75.

[21] Multari RA, Foster LE, Cremers DA, Ferris MJ. Effect of sampling geometry on elemental emissions in laser-induced breakdown spectroscopy. Applied Spectroscopy 1996;50:1483-99.

[22] Aguilera JA, Aragon C, Penalba F. Plasma shielding effect in laser ablation of metallic samples and its influence on LIBS analysis. Applied Surface Science $1998 ; 127-129: 309-14$.
[23] Bassiotis I, Diamantopoulou A, Giannoudakos A, Roubani-Kalantzopoulou F, Kompitas M. Effects of experimental parameters in quantitative analysis of steel alloy by laser-induced breakdown spectroscopy. Spectrochimica Acta Part B 2001;56:671-83.

[24] Aragon C, Aguilera JA, Penalba F. Improvements in quantitative analysis of steel composition by laser-induced breakdown spectroscopy at atmospheric pressure using an infrared Nd:YAG laser. Applied Spectroscopy 1999;53: 1259-67.

[25] Salle B, Cremers DA, Mauricec S, Wiensd RC, Fichet P. Evaluation of a compact spectrograph for in-situ and stand-off Laser-Induced breakdown spectroscopy analyses of geological samples on Mars missions. Spectrochimica Acta Part B 2005;60:805-15. 